\title{
Implementation of the Automatic and Interactive Chess Board
}

\author{
Allen R. Mendes, Atur M. Mehta, Bhavya H. Gohil \\ Department of Electronics Engineering, K.J Somaiya College of Engineering, Vidyavihar, Mumbai-India.
}

\begin{abstract}
This paper aims at throwing light on the new mode of playing board games by having an automated physical platform. Hence it discusses the development of an automatic chess board called as Chess.Automated. which enables the user to play the game of chess in different formats; with the opponents moves completely automated. It uses various electronic components such as the Arduino Mega2560 Microcontroller, Membrane Keypad and driver IC's along with different programming languages such C++, Python and Java to achieve automation between software and hardware.
\end{abstract}

Keywords: Arduino Mega2560, Chess.Automated, Online gameplay, Membrane keypad, MRL (My Robot Lab).

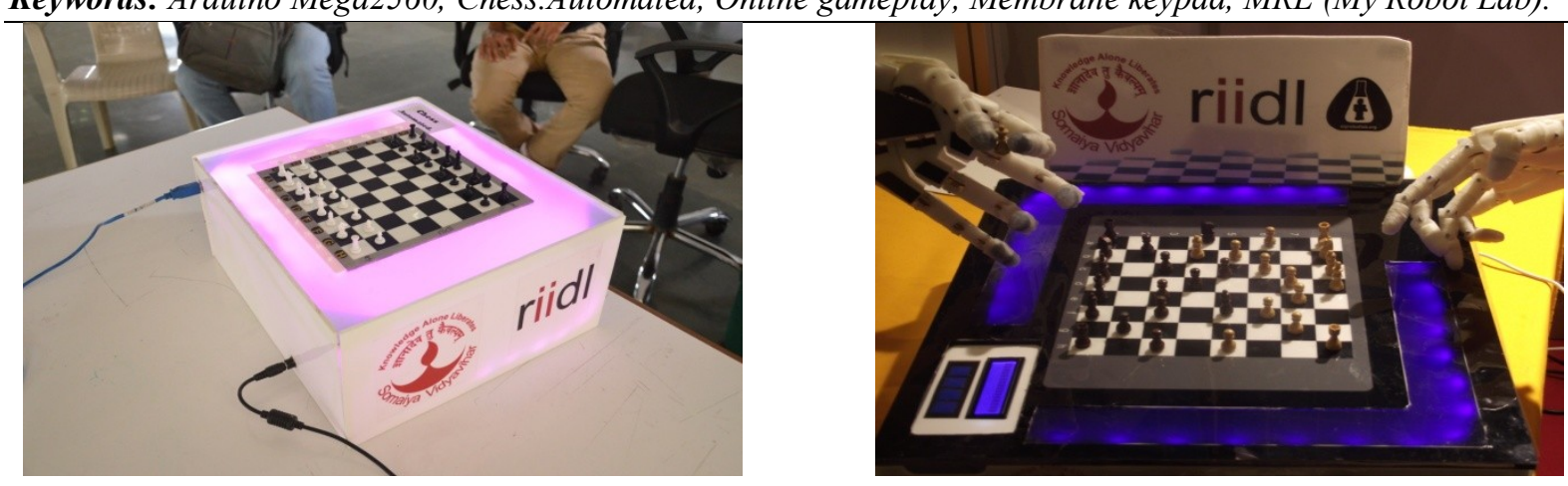

\section{Introduction}

With the technology moving a step forward, the world now prefers an interactive and real platform instead of stand-alone software's. Automating the famous board game: 'Chess' opens up a different avenue of implementing fiction into the reality of board games. This paper gives a detailed discussion on the creation of Chess.Automated. There are three modes of gameplay:

1. One player mode: In this mode, the user plays the game of chess against the computer on a physical board with the opponents moves completely automated. The user plays a move and then the opponent's chess piece physically moves on the chess board and positions itself on the desired location to represent the computer's move. Voice feedback of the move played is given by the computer along with attractive visual effects.

2. Two player mode: This mode enables the user to play the game of chess over the internet on a physical board. The user plays a move on his/her respective board (or on a web GUI) and this move is represented physically by dragging the particular chess piece on the opponent's chess board. Voice feedback of the move played is given by the computer along with attractive visual effects.

3. Chess for Blind: This is a novel feature of the Chess.Automated which enables a visually impaired person to play the game of chess against the computer or a human opponent on a physical board. This is made possible with features such as voice feedback of every move played, textural difference between white and black boxes and braille incripted chess pieces and co-ordinate system. Hence this would be the first physical platform by which a visually impaired person will be able to play the game of chess against the computer or against a human opponent over the internet.

\section{A. Arduino Mega ATmega 2560}

\section{Hardware Platforms}

The microcontroller used is Arduino Mega ATmega 2560. Arduino is an open source electronics prototyping platform which easy to use and program. It consists of 54 digital input/output pins and 16 analog pins out of which this project uses 45 pins. The function of microcontroller is to transfer data across the model i.e. it receives data from the sensing membrane keypad, communicates serially with the chess engine software and controls the X-Y plotter mechanism. It serially communicates with the computer at a baud rate of 9600 . 


\section{B. Sensing Membrane Keypad}

A $8 \times 8$ matrix keypad is used to simulate the 64 boxes on a chess board. It is $2 \mathrm{~mm}$ thick and is manufactured using Membrane Keypad technology which uses silver ink instead of wires. The 16 output pins are directly connected to the Arduino so as to get the co-ordinates of each move when the user presses the initial position keypad and then the final position keypad to register the played move.

\section{Hybrid Stepper Motor and X-Y plotter mechanism}

Stepper motor is a brushless DC motor which can be programmed to rotate a certain number of steps at any given time. The hybrid stepper motor is a combination of permanent magnet stepper motor and variable reluctance stepper motor. It is used because it works on a lower power rating $(12 \mathrm{~V})$ and provides higher speed and torque with minimal heating. These motors control the accurate movement of chess pieces over the board in the form of a co-ordinate system. For example: the X-axis stepper motor move the distance of one chess box from the origin in the $\mathrm{X}$-axis direction along with $\mathrm{Y}$-axis stepper motor moving one chess box from the origin in the $\mathrm{Y}$-axis direction, would in turn move a chess piece over the chess board to co-ordinates $(1,1)$. The $\mathrm{X}-\mathrm{Y}$ plotter mechanism is a rack and pinion arrangment attached to the stepper motors to move the center piece attachment (mounted with a servo motor) to each co-ordinate in the X-Y plane.

\section{Servo motor}

A Servo motor is a rotary actuator that allows for precise control of angular position with the help of an internal feeback control system. In this project the servo motor is used to couple and de-couple the magnets (one being on the tip of the servo lever and the other being underneath all the chess pieces). This action enables to drag the chess pieces over the board to represent physical movement of each move played.

\section{E. Motor and LED driver IC's}

The IC used for controlling the stepper motor is a dual H-bridge motor driver IC-L293D. Its low cost, consumes less power and is simple to interface 4 wire stepper motors. It has 4 input control pins and 4 output motor control pins. To drive the LED's, a Darlington pair IC-ULN2003 is used. An RGB LED strip oprating at $12 \mathrm{~V}$ is used for visual effects. The IC helps control the selection of the color of LED's to be displayed.

\section{Methodology}

The entire process is a two way cycle namely the input cycle and the output cycle. In the input cycle, the X-Y plotter mechanism sets itself to the origin (any one corner of the chess board) with the help of bump switches. Thereafter the positions of the chess pieces and the played move is registered and processed by the microcontroller and is then given in a particular format to chess engine software MRL (My Robot Lab) for calculating the best counter move according to the level of difficulty. The output cycle consists of the motor movements from the initial to final co-ordinates, servo action, voice and visual feedback. Also the online gameplay process is discussed in detail.

\section{A. Input cycle}

\section{Setting the origin}

The X-Y plotter mechanism requires a start point or origin so as to cover all the co-ordinates of the chess board in a defined and calculated number of steps of the stepper motor. For this, as soon as the board is powered on, the stepper motors move in the direction of the origin until they activate the bump switches. Bump or lever switches are used to detect physical contact between two surfaces. When the bump switches of both axes are activated, the setting up of the origin for the X-Y plotter mechanism is achieved.

\section{Detection of the played move co-ordinates and processing of data}

The sensing membrane keypad is a $8 \times 8$ matrix keypad and is continously scanned in the input cycle. It consists 8 pins for 8 rows and 8 pins for 8 columns. The process of scanning is done as follows:

- Pull one column to HIGH or $+5 \mathrm{~V}$ and read all the rows to find out the key pressed.

- Put a delay of $10 \mathrm{mS}$ for switch debounce.

- Pull the next column to $\mathrm{HIGH}$ or $+5 \mathrm{~V}$ and again read all the rows and repeat these steps for all the columns.

Once the initial and final co-ordinates of the played move are registered by the microcontroller, we then process the data into a suitable format so that the chess engine can understand the played move. The chess engine accepts data in the format: ( $\mathrm{x}$ initial.co-ordinate final.co-ordinate $\mathrm{n} / \mathrm{i} \mathrm{z}$ ). All these characters have a particular meaning. " $\mathrm{x}$ " is used to determine the start of the data string. Next comes the initial and final co- 
ordinates; for eg: a1a2 . "n" or " $\mathrm{i}$ " is used to determine the played move is valid or not where " $\mathrm{n}$ " stands for valid or normal move and " $\mathrm{i}$ " stands for invalid move. The chess engine won't accept any invalid moves. " $\mathrm{z}$ " is used to indicate the end of data string. Hence if the user plays a move from co-ordinates a1 to a2, the data processed by controller would be: xala2nz. This data is then given to the chess engine with the help of serial communication. This ends the input cycle.

\section{B. Output cycle}

\section{Stepper motor movements and servo action}

Once the the best counter move has been calculated by the MRL, it sends the co-ordinates through the serial communication port to the microcontroller. Now as the X-Y plotter mechanism is at the origin, the microcontroller calculates the number of steps required for both the $\mathrm{X}$-axis and $\mathrm{Y}$-axis stepper motors so that the mechanism reaches the desired co-ordinates. For example, the counter move's initial and final co-ordinates are $\mathrm{d} 2$ to $\mathrm{d} 4$. So the microcontroller will calculates the number of steps required to reach the "d" column and then the " 2 " row from the origin and the stepper motors move accordingly. After reaching $\mathrm{d} 2$ (initial position), the microcontroller commandes the servo to rotate 90 degrees so that the magnet attached to the servo lever tip engages with the the chess piece above the board. In this way the chess piece is now magnetically attached to the servo tip and will now move to any co-ordinate on the chess board the servo tip drags it to. Then the microcontroller calculates the number of steps required to move from $\mathrm{d} 2$ to $\mathrm{d} 4$ (to the "d" column and " 4 " row). The stepper motors rotates the calculated number of steps to reach $\mathrm{d} 4$, dragging the chess piece above it as the servo tip and the chess piece are still engaged to eachother. After reaching the final position, the microcontroller lower the servo to 0 degrees and this action dis-engages the magnetic servo tip and the chess piece, therby dragging a chess piece from co-ordinates $\mathrm{d} 2$ to $\mathrm{d} 4$.

\section{Voice and Visual feedback}

Every user action is attractively co-ordinated with visual and voice feedback. The colours are defined for each user action such:

- The lights display blue colour indicating the microcontroller is ready to accept the users input.

- The lights display white colour when the X-Y mechanism is setting itself to the origin.

- The lights display green colour when the computer is playing the counter move automatically.

- The lights display red colour when the user plays an invalid move or when the computer is killing the user's chess piece.

Voice feedback is given when the user completes his/her move. For example, if the user plays a pawn chess piece from $\mathrm{d} 2$ to $\mathrm{d} 4$, then the voice feedback service provided by MRL would give a voice output saying: "You played pawn from d2 to $\mathrm{d} 4$ ". In response to the users move, the counter move's voice feedback is also given by this service. This enables the visual impaired to understand the moves happening in the game through voice feedback. This ends the output cycle.

\section{Online gameplay}

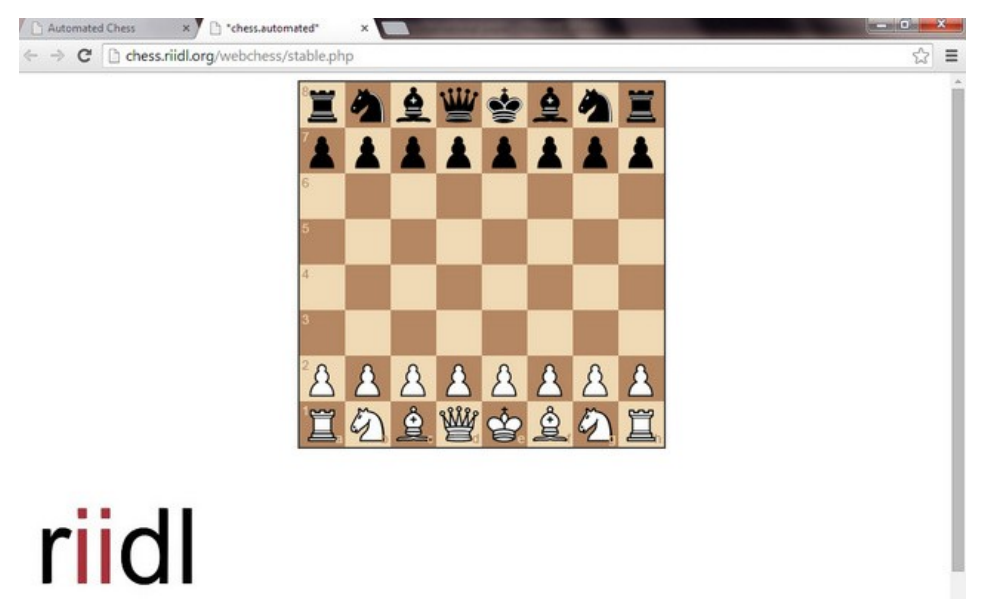

The automatic chess board can be connected to the internet and this enables any user to play the game of chess on a physical board over the internet. The user visits the website (www.chess.riidl.org) and selects the play new tab. This opens a web GUI of a chess board on the computer screen. After starting the game, the move played by the user on the physical board is represented on the web GUI as well as the opponent's automatic chess board in the form of physical movements of the chess piece. A php script is used to obtain the played move by user A through the serial communication port and is transferred over the internet and given to user B's 
server, which is then processed by the microcontroller to achieve physical movements of the chess pieces. For example, user A plays white pawn from $\mathrm{d} 2$ to $\mathrm{d} 4$ on his/her chess board, this move is represented on the web GUI as well as the white pawn on user B's automatic chess board would move from $\mathrm{d} 2$ to $\mathrm{d} 4$. The same process applies when user B plays any move. This feature enables any user (even the visually impaired) to play the game of chess against any user in the world on a physical board.

\section{Discussions And Conclusion}

The main aim of this project was to create an automatic chess board for the visually impaired with voice feedback and to retain the feel of board games in a world where software gaming has taken over the physical feel of games and sports. Also enabling the user to play board games on a physical board over the internet was achieved. Certain aspects in this paper are currently been worked on to improve the project entirely and add more features to it. The future model would have an LCD to display commands and instructions, belt and pulley mechanism for faster movement of chess pieces, on board processor, voice controlled gameplay, mode selection (online, offline and learning chess) and compact design.

In summary, this paper discuses the automation of board games and how this method can be used to automate any board game or create an automated X-Y plotter mechanism for various purposes like laser cutting machine, CNC, 3D printer and graph plotter.

\section{Acknowledgment}

This project was supported by Riidl (www.riidl.org) and Somaiya Vidyavihar (www.somaiya.edu), Mumbai-India.

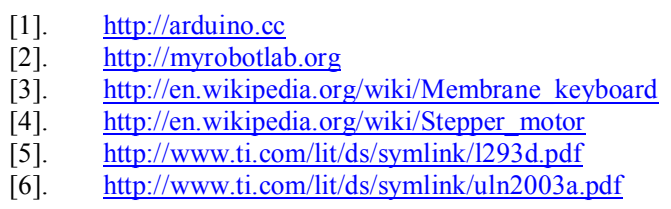

\section{References}

